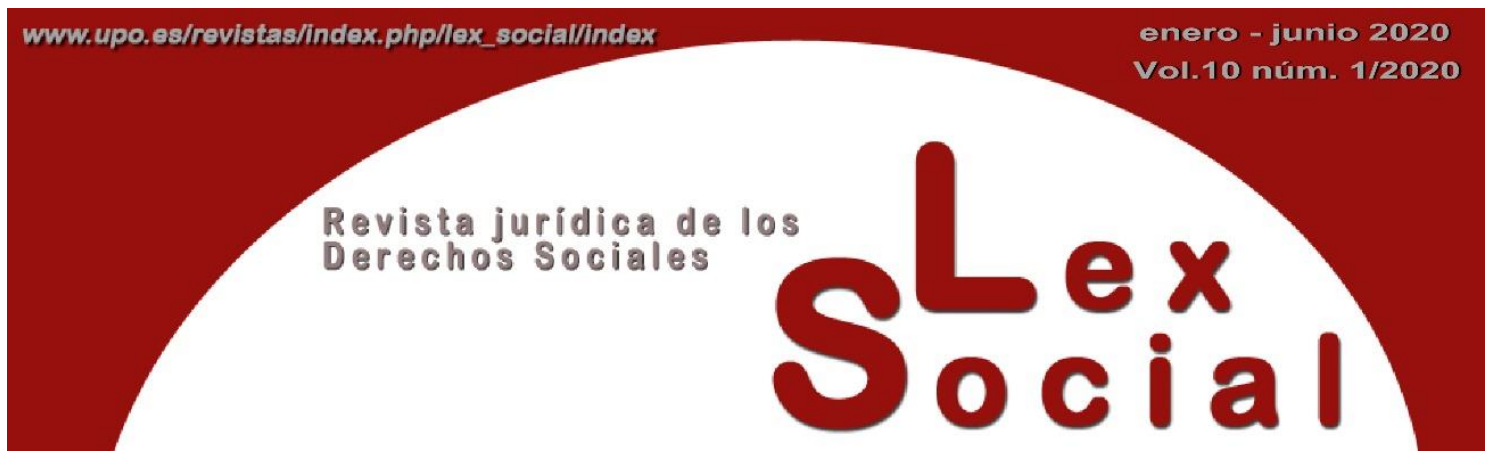

\title{
LA ERRADICACIÓN DE LA POBREZA: UNA CUESTIÓN DE DERECHOS CIVILES
}

\section{THE ERADICATION OF POVERTY: A CIVIL RIGHTS ISSUE}

\author{
RICARDO GARCÍA MANRIQUE \\ Profesor Titular de Universidad \\ Universidad de Barcelona
}

Artículo recibido el 16 de octubre de 2019

Artículo aceptado el 14 de noviembre de 2019

\section{RESUMEN}

La pobreza constituye una violación de los derechos civiles. Una consecuencia jurídica práctica e inmediata de esta idea es que quienes sean titulares de los derechos civiles y estén en situación de pobreza han de disponer de una acción jurisdiccional para reclamar que esa situación suya sea remediada. Para sostener esa idea me apoyaré en cierta línea jurisprudencial contemporánea constituida por una serie de decisiones de tribunales nacionales e internacionales.

Con carácter preliminar, haré una referencia a la Agenda 2030 y otra a la labor de los defensores del pueblo, con la intención de hacer ver: (1) que la idea que pretendo defender aquí puede contribuir a fortalecer la legitimidad y la eficacia de los objetivos de la Agenda; y (2) que se trata de una idea útil para la labor de las instituciones de defensa de los derechos humanos.

Palabras Clave: Pobreza, derechos civiles, Agenda 2030, defensor del pueblo.

\section{Abstract}

Poverty is a violation of civil rights. A practical and immediate legal consequence of this idea is that those who hold civil rights and are in a situation of poverty must have a jurisdictional action to demand that their situation be remedied. To support 
this idea, I will rely on a recent judicial tendency made up of a series of decisions issued by national and international courts.

On a preliminary basis, I will make a reference to the 2030 Agenda and another to the work of the ombudsmen, with the intention of making it appear: (1) that the idea that I intend to defend here can contribute to strengthening the legitimacy and effectiveness of the Agenda objectives; and (2) that this is a useful idea for the work of human rights defense institutions.

KEYWORDS: Poverty, civil rights, 2030 Agenda, ombudsman.

\section{SUMARIO}

1. La Agenda 2030, la pobreza y los derechos.

2. Las defensorías del pueblo y el lenguaje de los derechos.

3. El mínimo vital: una cuestión de derechos civiles.

4. Conclusiones.

\section{La Agenda 2030, la pobreza y los derechos}

La Agenda 2030 para el Desarrollo Sostenible fue aprobada por la Asamblea General de la ONU el 25 de septiembre de 2015. Establece 17 objetivos de desarrollo sostenible que deberían ser alcanzados en los próximos 15 años. Aunque el contenido de estos objetivos es bastante diverso, podemos decir que el principal de todos ellos es la erradicación de la pobreza en el mundo. En efecto, el Objetivo 1 es "poner fin a la pobreza en todas sus formas y en todo el mundo" y con él se relacionan de manera directa o indirecta muchos otros objetivos, relativos al hambre, la vida sana, la educación, el agua, la energía, el crecimiento económico o las infraestructuras. A mayor abundamiento, ya en el primer párrafo del Preámbulo se puede leer que "la erradicación de la pobreza en todas sus formas y dimensiones, incluida la pobreza extrema, es el mayor desafío a que se enfrenta el mundo".

Se ha dicho que la Agenda "no utiliza el lenguaje de los derechos" (Queralt y Seleme 2018: 408), sino el de los "objetivos", esto es, la Agenda no establece como fin la garantía de ciertos derechos sino la consecución de ciertos objetivos. Es verdad que los derechos humanos son aludidos en varios párrafos de la Declaración preliminar (aparecen expresamente, por lo menos, en el 3, el 7, el 8, el 10, el 19 y el 20); pero también lo es que, a continuación, a la hora de fijar los fines de la Agenda, se recurre, 
en efecto, al lenguaje de los objetivos. En principio, esta opción tiene consecuencias, porque "derechos" y "objetivos" son estándares normativos distintos. Dicho de manera muy simple, los derechos son jurídicamente exigibles, pero los objetivos lo son sólo políticamente. Quien tiene un derecho puede exigir su satisfacción ante un tribunal; en cambio, quien se podría beneficiar de la consecución de un objetivo sólo puede censurar políticamente su frustración. Sin embargo, esto no significa que la opción de la Agenda por el lenguaje de los objetivos sea censurable. De hecho, hay quienes entienden que este lenguaje es más apropiado para los fines pretendidos, por varias razones: (1) definen metas cuantificables; (2) están sujetos a plazos específicos en vez de invocar la "realización progresiva", como en el caso de los derechos socioeconómicos; y (3) gozan de mayor apoyo que los derechos humanos ${ }^{1}$ (Queralt y Seleme 2018: 407-408).

En todo caso, a pesar del lenguaje utilizado, Jahel Queralt y Hugo Seleme sugieren "anclar la Agenda en los derechos humanos", revistiendo "la primera de la autoridad legal que caracteriza a los segundos". Este anclaje tendría, al menos, tres consecuencias: en primer lugar, el fracaso en la consecución de los objetivos de la Agenda habría de ser interpretado como un incumplimiento de derechos, lo cual abriría la puerta a potenciales recursos ante tribunales nacionales o internacionales; en segundo lugar, la principal responsabilidad en el cumplimiento de la Agenda se asignaría a cada uno de los estados respecto de su población y territorio, y no a terceros estados o a organizaciones internacionales (es decir, se trata de adoptar una perspectiva estadocéntrica, que es la propia de la garantía de los derechos humanos, en vez de una donantecéntrica, más vinculada con los programas de ayuda internacional al desarrollo); en tercer lugar, los indicadores de la Agenda podrían ser utilizados como métrica de la consecución de los derechos socioeconómicos, de cara a determinar el grado de cumplimiento de estos derechos por parte de los estados (Queralt y Seleme 2018: 409-410).

Siguiendo esta indicación, de lo que se trata es de precisar de qué modo tendría lugar este anclaje de la Agenda en los derechos. En el trabajo citado en los párrafos anteriores parece que las medidas de lucha contra la pobreza se vinculan con la ejecución de derechos sociales. En cambio, aquí trataremos de ensayar otro camino.

\section{Las defensorías del pueblo y el lenguaje de los derechos}

Recurrir al lenguaje de los derechos en vez de al lenguaje de los "objetivos" y "metas" de carácter socioeconómico puede ser muy útil para la labor de las defensorías del

\footnotetext{
${ }^{1}$ Por ejemplo, tanto China como Estados Unidos se han comprometido con la Agenda 2030, pero se han negado, respectivamente, a suscribir el Pacto Internacional de Derechos Civiles y Políticos y el Pacto Internacional de Derechos Económicos, Sociales y Culturales.
} 
pueblo. La razón es muy sencilla: las defensorías son instituciones creadas al servicio de la garantía y promoción de los derechos humanos. Por eso, si las defensorías quieren comprometerse con los objetivos de la Agenda 2030, y en particular con la lucha contra la pobreza, harán bien en traducir esos objetivos en derechos. En consecuencia, siendo posible (y creo que lo es), la participación de las defensorías en la lucha contra la pobreza ha de concebirse como un empeño por garantizar derechos humanos, y eso requiere justificar que la pobreza es, en efecto, una violación de derechos humanos.

Tradicionalmente, quienes han vinculado la pobreza con los derechos humanos lo han hecho con los derechos sociales (o, si se quiere, con los derechos "económicos, sociales, culturales y ambientales", aunque soy partidario de esa otra denominación más simple). Este vínculo resulta obvio y no requiere explicación. Sin embargo, en términos de eficacia, el problema que suscita es que los derechos sociales todavía no han sido plenamente aceptados como derechos humanos, o no se admite que su rango normativo sea el mismo que el de los demás derechos. Si nos fijamos en el plano estatal, que es el que acaso interesa más a instituciones igualmente estatales como son las defensorías del pueblo, muchas constituciones no atribuyen el mismo estatuto a todos los derechos, siempre con desventaja para los derechos sociales, que, con frecuencia, no son considerados derechos genuinos. Por poner un solo ejemplo, en la Constitución Española, muchos de esos derechos son considerados "principios rectores de la política social y económica", y buena parte de la doctrina entiende, con buenas razones (aunque discutibles), que eso significa que no son auténticos "derechos fundamentales", con serias consecuencias en materia de garantías, mucho menores para los derechos sociales que para los civiles y políticos. Guste o no guste, de momento esto es así, e insisto en que se trata de una pauta generalizada en el derecho constitucional comparado. Frente a este hecho, y con el fin de desvirtuar su relevancia, entiendo que no cabe objetar la tesis de la "indivisibilidad" de los derechos: en primer lugar, porque creo que esta tesis, bien entendida, no niega la distinción entre derechos civiles y derechos sociales, sino que la matiza (véase, por ejemplo, Nickel 2008, donde se contiene un ejercicio de refinamiento del significado de dicha tesis; por otra parte, son muchos los análisis académicos que demuestran que las diferencias entre derechos civiles y sociales no son tan profundas como se ha querido hacer ver; sin embargo, a mi juicio, de entre esos análisis, los más esclarecidos no son los que niegan la distinción, sino los que la reducen; un ejemplo de ello se encuentra en Hierro 2007). En segundo lugar, porque la tesis de la indivisibilidad podría ayudar a poner en cuestión la justicia del distinto tratamiento que unos y otros derechos reciben, pero no el hecho mismo de que reciban ese distinto tratamiento.

Por eso, si la pobreza pudiera concebirse como una violación no ya de derechos sociales, sino de derechos civiles, sería mucho más fácil construir un argumento jurídico a favor de su erradicación, puesto que los derechos civiles sí gozan de la 
máxima protección jurídica en cualquier estado constitucional. De este modo, las defensorías del pueblo estarían mucho más legitimadas para participar en esa lucha contra la pobreza, ejerciendo la acción que les resulta más característica o definitoria: la defensa de los derechos humanos garantizados por la Constitución. Por supuesto, no ha de encontrarse aquí un argumento contra los derechos sociales basado en eso que se llama "la fuerza normativa de lo fáctico", ni siquiera una asunción más o menos resignada del statu quo constitucional. Me limito a sostener que, a día de hoy, el combate contra la pobreza puede tener más éxito si se entabla en el nombre de los derechos civiles que si lo hacemos en el nombre de los derechos sociales.

Hay, además, una segunda razón que anima a desvincular la lucha contra la pobreza de los derechos sociales. Es la siguiente: con esa desvinculación contribuimos a evitar el riesgo de rebajar la ambición normativa de los derechos sociales, un riesgo al que vienen sometidos durante las últimas décadas como consecuencia, diría, de la crisis actual del pensamiento socialista que había ido dando forma a los derechos sociales a partir de mediados del siglo XIX. De acuerdo con esta forma de concebirlos, el objetivo de los derechos sociales era la eliminación de la desigualdad social o, como me gusta llamarlo, la puesta en práctica de "la libertad de todos". Con la reivindicación de estos derechos no se trataba, pues, de limitarse a luchar contra las formas más groseras de la desigualdad (en una palabra: contra la pobreza), sino de algo mucho más ambicioso: la eliminación de las posiciones sociales desiguales respecto de todo aquello considerado relevante para una vida buena (el trabajo, la educación, la asistencia). De hecho, la consideración de la erradicación de la pobreza como un objetivo irrenunciable es ya propia de las primeras etapas del pensamiento político y jurídico moderno, de orientación contractualista. La encontramos ya en Hobbes o en Locke; y determinadas cláusulas de las primeras constituciones y declaraciones de derechos así lo reconocían también. La idea, en síntesis, es que ninguna comunidad política puede ser legítima si no garantiza la "existencia" o la "subsistencia" digna de todos sus miembros, esto es, si permite que algunos de ellos sean "pobres". En cambio, los derechos sociales, tal y como fueron configurados más tarde, expresan una exigencia mucho mayor: la de que todos tengamos igual acceso a los bienes básicos necesarios para llevar adelante una vida buena; y, como consecuencia, la exigencia de que las actividades sociales fundamentales (de nuevo, el trabajo, la educación, la asistencia) se desarrollen en condiciones igualitarias que eviten la subordinación de unos respecto de otros. Tal como lo dejó escrito T. H. Marshall en 1950:

"La reducción de las diferencias de clase es aún la meta de los derechos sociales, pero ha adquirido un nuevo significado, porque no se trata sólo de acabar con la miseria obviamente desagradable de los estratos más bajos de la sociedad, sino que se ha transformado en un conjunto de actos que modifican el modelo global de la desigualdad. Ya no basta con elevar el nivel más bajo 
del edificio social, dejando intacta la superestructura. Ahora se ha comenzado a remodelar todo el edificio" (Marshall 1992 [1950]: 52).

Sin embargo, la vinculación de los derechos sociales con una empresa desde luego muy loable, pero mucho más modesta como es la erradicación de la pobreza, puede implicar, como decía, una mengua de la fuerza emancipatoria (o igualatoria) de los derechos sociales, o incluso su desnaturalización. Esto no le parecerá mal, o no contrario a su naturaleza, a quien sostenga una concepción "liberal" de los derechos sociales, acorde con sus primeras etapas de desarrollo y seguramente con la tendencia observable en los últimos tiempos, una concepción según la cual los derechos sociales son derechos "a un mínimo" cuyo enemigo no es la desigualdad sino (sólo) la pobreza (Atria 2004: 31-32). En cambio, a mi juicio, esa opción por una concepción liberal y mínima de los derechos sociales supone dar un paso atrás y privarlos (a ellos y a los derechos humanos en general) del estatuto de criterio máximo de legitimidad política

y jurídica de que gozan ahora. Pues si los derechos humanos dejan de anunciar una comunidad igualitaria y se limitan a prometer simplemente la supresión de las formas más groseras de la desigualdad habrán perdido mucho del atractivo del que gozan todavía.

Son, por tanto, estas dos razones (la mayor fuerza normativa de los derechos civiles y la conveniencia de reservar los derechos sociales para objetivos igualitarios más ambiciosos) las que hacen preferible concebir la erradicación de la pobreza como una cuestión de derechos civiles, siendo esto posible. Veremos a continuación de qué manera lo han justificado algunos tribunales nacionales e internacionales.

\section{El mínimo vital: una cuestión de derechos civiles}

Desde principios de la década de los noventa, es posible identificar una serie de decisiones judiciales nacionales e internacionales que abogan por atribuir a todos los ciudadanos el derecho a la satisfacción de un "mínimo vital", esto es, de ciertas condiciones socioeconómicas básicas relativas a la alimentación, el alojamiento, la asistencia sanitaria o incluso la educación. Se trata de condiciones que, de ser satisfechas para todos, supondrían la erradicación de la pobreza (al menos, de la pobreza extrema) y con ello la consecución del objetivo último de la Agenda 2030. Esto es, en términos de derechos, la garantía del derecho a un mínimo vital sería el correlato más ajustado de tal objetivo. La base normativa de estas decisiones es diversa e incluye valores, principios y derechos de distinto tipo, aunque quiero llamar la atención sobre el hecho de que, a pesar de que en ocasiones los derechos sociales forman parte de esa base, acaso no sean necesarios para justificar el derecho al mínimo vital y baste con vincularlo o bien con algún derecho civil o bien con las condiciones básicas necesarias para asegurar el disfrute de todos los derechos. Conviene recordar 
que, en las jurisdicciones implicadas, el derecho al mínimo vital no es un derecho establecido de manera explícita; por eso, ha debido ser construido a partir de otras disposiciones normativas de orden constitucional.

Un derecho semejante sí cabe encontrarlo, por el contrario, en normas internacionales de derechos humanos. En particular, el art. 25.1 de la Declaración Universal de los Derechos Humanos establece:

"Toda persona tiene derecho a un nivel de vida adecuado que le asegure, así como a su familia, la salud y el bienestar, y en especial la alimentación, el vestido, la vivienda, la asistencia médica y los servicios sociales necesarios; tiene asimismo derecho a los seguros en caso de desempleo, enfermedad, invalidez, viudez, vejez u otros casos de pérdida de sus medios de subsistencia por circunstancias independientes de su voluntad".

O también el art. 11.1 del Pacto Internacional de Derechos Económicos, Sociales y Culturales, correlato del anterior:

"Los Estados Partes en el presente pacto reconocen el derecho de toda persona a un nivel de vida adecuado para sí y su familia, incluso alimentación, vestido y vivienda adecuados, y a una mejora continua de las condiciones de existencia".

Sin embargo, cabe observar que en estos preceptos no se contempla un derecho "al mínimo vital", sino algo más exigente y probablemente de más difícil justiciabilidad, como es el "nivel de vida adecuado", que, además, parece que habría de progresar de manera indefinida (es decir, acaso se parece más a un "objetivo" que a un "derecho"). Sea como sea, dejaremos de lado estas normas de derecho internacional "universal" para centrarnos en esa línea jurisprudencial en la que se incluyen decisiones de tribunales superiores nacionales y otras de tribunales internacionales regionales de derechos humanos.

En 1992, la Corte Constitucional colombiana (Sentencia T-426/92) reconoció un "derecho al mínimo vital", basado en los principios de dignidad y de Estado social de Derecho. El derecho resultaba configurado en términos muy amplios, puesto que "no sólo incluye la facultad de neutralizar las situaciones violatorias de la dignidad humana, o la de exigir asistencia y protección por parte de personas o grupos discriminados, marginados o en circunstancias de debilidad manifiesta (...), sino que, sobre todo, busca garantizar la igualdad de oportunidades y la nivelación social en una sociedad históricamente injusta y desigual" ( $\left.\mathrm{FJ} \mathrm{n}^{\circ}{ }^{6}\right)$. La Corte añadía que de aquí no había de seguirse un derecho subjetivo para toda persona "a una prestación económica 
del Estado", "sin atender a las especiales circunstancias del caso". Por eso, en la sentencia, examinó esas circunstancias y llegó a la conclusión de que el estado de debilidad manifiesta del anciano recurrente y la incapacidad material de la familia para asistirlo justificaban la titularidad de un derecho subjetivo de aplicación inmediata (véase Champeil Desplats 2012: 229).

En 1995, el Tribunal Federal suizo reconoció un derecho equivalente como derecho subjetivo de todos los ciudadanos, considerado como la "condición de ejercicio de todos los demás derechos fundamentales". Pocos años después, en 1999 y en consonancia con dicha decisión, la Constitución suiza incorporó este derecho en su artículo 12:

"Cualquiera que esté en una situación de apuro [détresse] y no esté en condiciones de subvenir a su subsistencia tiene el derecho a ser ayudado y asistido, y a recibir los medios indispensables para mantener una existencia conforme con la dignidad humana".

En años subsiguientes, otros tribunales superiores han reconocido derechos muy similares. Es el caso del Tribunal Supremo de la India (a partir de 1996), de Israel (reiteradamente a partir de 1998), de Sudáfrica (2004), y del Tribunal Constitucional Federal alemán (2010) (sobre todas estas decisiones, véase Fercot 2012 y Roman 2012, donde se contienen análisis más pormenorizados que el que se ofrece a continuación a modo de síntesis).

Sobre el conjunto de estas decisiones cabe decir todas ellas han atribuido a los ciudadanos de los países implicados un derecho al mínimo vital, en unos u otros términos. Se trata, siempre, de respuestas judiciales a situaciones de extrema pobreza que, si no son resueltas, resultan incompatibles con cualquier pretensión de legitimidad por parte de la autoridades políticas, un punto en el que todos los tribunales insisten con mayor o menor vehemencia. El fundamento del derecho al mínimo vital varía de unas a otras decisiones, aunque con carácter general cabe afirmar que se hace radicar en nociones capitales de toda teoría de los derechos fundamentales (dignidad, autonomía, integridad personal, vida, carácter social del Estado). La Corte Constitucional colombiana se apoya, como hemos visto, en los principios de dignidad y Estado social; el Tribunal Supremo de Israel lo hace en la misma idea de dignidad; el Tribunal Constitucional alemán también recurre al principio del Estado social, tan fecundo en su jurisprudencia; el Tribunal Supremo de la India recurre a una interpretación extensiva del derecho a la vida; y el Tribunal Supremo de Sudáfrica, en fin, combina las ideas de dignidad y de derecho a la vida (Fercot 2012: 60ss.). En ninguna de estas decisiones ha sido necesario recurrir a los "derechos sociales", aunque resulten aludidos en algunas de ellas. Sí al principio del Estado social, en los 
casos colombiano y alemán, pero obsérvese que de este principio, muy abstracto, no se sigue necesariamente la existencia de derechos sociales concretos (aunque véanse las consideraciones sobre la relación entre el "Estado social" y los "derechos sociales" contenidas en Escobar 2012: 374-376).

El derecho al mínimo vital resulta más claramente vinculado con un derecho civil concreto en el caso de los pronunciamientos del Tribunal Europeo de Derechos Humanos (TEDH) y de la Corte Interamericana de Derechos Humanos (CIDH). En particular, se trata del derecho a la integridad física y moral, especificado o no en la prohibición de tratos degradantes. Es cierto que ninguno de los dos ha afirmado con claridad la justiciabilidad de un derecho al mínimo vital en sus respectivos sistemas regionales. Sin embargo en la jurisprudencia de ambos se encuentran mimbres suficientes para urdir ese derecho sin mucha dificultad.

En el caso del TEDH no encontramos todavía una sentencia que conceda el derecho al mínimo vital, pero sí una decisión de inadmisión en la cual el Tribunal dejó sentado cuál sería el fundamento de este derecho:

"El Tribunal considera que una queja relativa al importe insuficiente de una pensión o de otros beneficios sociales puede, en principio, plantear una cuestión que caiga bajo el artículo 3 del Convenio, que prohíbe los tratos inhumanos o degradantes" (Larioshina c. Rusia, 23 de abril de 2002, decisión de inadmisión).

Es cierto que, en este caso (como en el muy similar de Budina c. Rusia, 18 de junio de 2009, donde se encuentra una apreciación similar), el Tribunal de Estrasburgo decidió no admitir la demanda, porque juzgó que los medios de que disponía la demandante la situaban por encima del umbral de pobreza que marca el límite del trato inhumano o degradante; pero lo que importa es que el Tribunal aceptó que, si la pobreza es realmente extrema, podemos estar ante una situación prohibida por el Convenio, una situación que los Estados, por tanto, tienen la obligación de remediar (López Guerra 2015: 404-405; Mestre 2016: 116). De todas formas, su propia jurisprudencia posterior en relación con asuntos similares todavía no permite afirmar con rotundidad que el Tribunal europeo haya decidido proteger el derecho a un mínimo vital (Nivard 2012: 518 ss.). Cabe insistir, aun así, en que el fundamento para un derecho semejante lo encuentra el Tribunal en el articulado del Convenio, un texto que, como bien se sabe, no recoge derechos sociales, sino sólo derechos civiles y políticos. Una pieza esencial del razonamiento del Tribunal es que las autoridades pueden infligir por omisión un trato inhumano o degradante de los prohibidos por el art. 3 del Convenio. O, lo que es lo mismo, que los derechos civiles y políticos establecidos en el Convenio generan, para las autoridades, no sólo obligaciones negativas (u omisiones) sino obligaciones 
positivas (o acciones), tal como el TEDH viene sosteniendo por lo menos de desde la sentencia Airey c. Irlanda, de 1979. De aquí que el incumplimiento de una de esas obligaciones positivas pueda ser constitutiva de la violación de un derecho civil, como es, en este caso, la prohibición de los tratos inhumanos o degradantes.

Por su parte, la Corte Interamericana de Derechos Humanos ha dictado dos sentencias en las cuales reconoce el derecho a un mínimo vital de la población reclusa, en este caso derivado del derecho a la integridad física y moral que está recogido en el art. 5 de la Convención Americana de Derechos Humanos. Se trata de las sentencias Yvon Neptune vs. Haiti (2008) y de Pacheco Teruel y otros vs. Honduras (2012), la primera relativa a las condiciones de encarcelamiento del expresidente de Haití, Yvon Neptune, y la segunda suscitada por la muerte de más de un centenar de reclusos en el incendio de una cárcel de San Pedro Sula, en la que se acreditaron unas condiciones de seguridad e higiene deplorables. Tres observaciones ahora son pertinentes en relación con estas dos decisiones.

En primer lugar, la jurisprudencia de la CIDH ha acreditado la existencia de un derecho a la vida humana digna (equivalente al derecho al mínimo vital) en el seno del sistema interamericano de derechos humanos. Este derecho se ha atribuido a las personas privadas de libertad, y encuentra su fundamento directo en los arts. 5.1 y 5.2 de la Convención Americana de Derechos Humanos, unido a la posición especial de garante que corresponde a las autoridades públicas frente a personas sometidas a su control. Sus contenidos principales son ciertos niveles mínimos (o decentes) de alojamiento, agua y vivienda, a los que cabría añadir otros sin demasiado esfuerzo argumentativo.

En segundo lugar, parece posible extrapolar este derecho a todas las personas, más allá del ámbito penitenciario, siempre que se admita que el trato inhumano (y el consiguiente menoscabo de la integridad personal) consiste en la privación de tales niveles mínimos de vida, con independencia del entorno (penitenciario o no) en que se padezca (esto es, interpretando la cláusula de la "posición de garante" en un sentido amplio, o bien simplemente no considerándola determinante para fundar el argumento de la Corte); y siempre que se admita (como lo hace el TEDH en el caso europeo) que los tratos inhumanos pueden ser causados por omisión, o que, en este contexto, la distinción entre acciones y omisiones carece de significado y, por tanto, deviene irrelevante.

En tercer lugar, este derecho a la vida humana digna no sería un derecho autónomo, sino la expresión de ciertos estándares vitales por debajo de los cuales no cabe garantizar el derecho a la integridad. Se trata, por tanto, de garantizar el derecho (civil) a la integridad y no de garantizar ciertos derechos sociales (agua y alimentación son los ejemplos más claros) incluidos en el Protocolo de San Salvador y, de acuerdo con 
él, no justiciables (aunque esto puede ser discutible, y de hecho ha sido discutido, no lo haremos aquí; véase, por ejemplo, Mejía 2016: 157 y Ferrero 2016: 1005). Estos derechos (sociales), en tanto que fundamentales, requieren una apropiación comunitaria y una distribución igualitaria de los recursos implicados que el sistema interamericano de derechos humanos considera de realización progresiva y no justiciable. La CIDH, en tanto que justifique el derecho a la vida humana digna a través del derecho a la integridad personal, y en tanto que le atribuya sólo ciertos contenidos mínimos, no se extralimita y cumple fielmente con su mandato.

\section{Conclusiones}

Una visión conjunta de las decisiones judiciales nacionales e internacionales a las que hemos hecho referencia permite observar algunas características comunes a todas ellas que, a su vez, nos habilitan para identificar de manera más o menos unitaria un "derecho al mínimo vital":

- El fundamento del derecho al mínimo vital se encuentra en distintas combinaciones de un pequeño grupo de derechos y principios: dignidad, autonomía, integridad, vida y estado social. Que se opte por una combinación u otra suele estar en función de las normas constitucionales (o internacionales) a las que puede recurrir cada tribunal.

- El derecho al mínimo vital no es, por tanto, un derecho autónomo, sino un derecho conexo con otros o integrado en otros.

- En todo caso, no se trata de un "derecho social" ni su justificación requiere invocar este tipo de derechos. Por el contrario, cuando se ha invocado un derecho concreto, ha sido el de la integridad física y moral y la correlativa prohibición de los tratos inhumanos o degradantes, esto es, un derecho civil clásico. En otras ocasiones se ha entendido que la satisfacción de este derecho es condición del ejercicio de todos los demás derechos, o que deriva de los principios generales inspiradores del conjunto de todos los derechos fundamentales.

- El contenido del derecho incluye, con ligeras variaciones, ciertos mínimos de alimentación, agua, vestido, alojamiento, electricidad y salud. Esta diversidad de contenidos refleja el hecho de que la pobreza extrema genera precariedades de todo tipo.

- La titularidad se considera universal, pero la condición de aplicación del derecho es la situación de pobreza extrema, una situación que es considerada como excepcional, aunque esta excepcionalidad, desde luego, está en función de las condiciones socioeconómicas de cada país.

- El derecho al mínimo vital es un derecho directamente justiciable por los tribunales, en la medida en que se funda en otros derechos o principios también 
justiciables $\mathrm{y}$ en que responde a situaciones particularmente graves que requieren una respuesta urgente.

- En definitiva, las defensorías del pueblo bien pueden invocar esta jurisprudencia, rica y diversa (y, en el caso de las latinoamericanas, específicamente la de la CIDH), para justificar que la pobreza (al menos, la pobreza extrema) atenta contra los derechos civiles de las personas (contra todos ellos en conjunto; y, en particular, contra el derecho a la integridad) y que el objetivo prioritario de la Agenda 2030, la erradicación de la pobreza, es por tanto una cuestión de derechos humanos.

\section{Bibliografía}

Atria, Fernando (2004): “¿Existen derechos sociales?”, en Discusiones 4: 15-59.

Champeil Desplats, Véronique (2012): "La justiciabilité des droits sociaux en Amérique Latine", en D. Roman (dir.), La justiciabilité des droits sociaux : vecteurs et résistences, París, Pedone: 223-235.

Escobar Roca, Guillermo (2012): "El Estado social”, en G. Escobar Roca (dir.), Derechos sociales y tutela antidiscriminatoria. Cizur Menor, Aranzadi: 363-376.

Fercot, Céline (2012) "Le juge et le droit au minimum. Les ambigüités du droit à des conditions minimales d'existence en droit comparé”, en D. Roman (dir.), La justiciabilité des droits sociaux : vecteurs et résistences, París, Pedone: 49-65.

Ferrero, Julie (2016): “État des lieux de la justiciabilité des droits économiques, sociaux et culturels dans le système interaméricain”, en Revue Trimestrielle des Droits de l'Homme 108: 983-1005.

Hierro, Luis L. (2007): "Los derechos económico-sociales y el principio de igualdad en la teoría de los derechos de Robert Alexy”, en R. Alexy et al., Derechos sociales y ponderación. Madrid, Fundación Coloquio Jurídico Europeo: 163-222.

López Guerra, Luis (2015): “Crisis económica y derechos humanos. Una nota de jurisprudencia”, en Teoría y realidad constitucional 36: 399-414.

Marshall, T. H. (1992 [1950]): Ciudadanía y clase social. Madrid, Alianza.

Mejía Rivera, Joaquín A. (2016): “Los derechos económicos, sociales y culturales a la luz del sistema interamericano de derechos humanos", en L. E. Ríos Vega e I. Spigno (dirs.), Estudios de casos líderes interamericanos y europeos. México, Tirant lo Blanch: 127-158.

Mestre, Ruth (2016): "La protección de los derechos sociales por el Tribunal Europeo de Derechos Humanos", en Cuadernos Electrónicos de Filosofía del Derecho 33: 113132. 
Nickel, James W. (2008): "Rethinking Indivisibility: Towards a Theory of Supporting Relations between Human Rights", en Human Rights Quarterly, 30: 984-1001.

Nivard, Carole (2012): La justiciabilité des droits sociaux. Étude de droit conventionnel européen. Bruselas, Bruylant.

Queralt, Jahel y Seleme, Hugo (2018): "La agenda para el desarrollo y los derechos humanos: instrumentos complementarios”, en Anuario de Filosofía del Derecho XXXIV: 403-422.

Roman, Diane (2012): "Les droits civils au renfort des droits sociaux. L'interchangeabilité des droits fondamentaux dans le discours judiciaire", en La Revue des droits de l'homme 1: 319-338. 